\title{
An improved velocity increment model based on Kolmogorov equation of filtered velocity
}

\author{
L. Fang, ${ }^{1,2}$ L. Shao, ${ }^{1,2}$ J. P. Bertoglio, ${ }^{1,2}$ G. X. Cui, ${ }^{3}$ C. X. Xu, ${ }^{3}$ and Z. S. Zhang ${ }^{3}$ \\ ${ }^{1}$ Laboratoire de Mécanique des Fluides et d'Acoustique, École Centrale de Lyon, \\ 69130 Ecully, BP 163, France \\ ${ }^{2}$ CNRS, INSA de Lyon, Université de Lyon, Université Lyon 1, France \\ ${ }^{3}$ Department of Engineering Mechanics, Tsinghua University, 100084 Beijing, China
}

(Received 23 September 2008; accepted 12 May 2009; published online 15 June 2009)

\begin{abstract}
The velocity increment (VI) model, which was introduced by Brun et al., is improved by employing the Kolmogorov equation of filtered velocity in this paper. This model has two different formulations: a dynamic formulation and a simplified constant form in high Reynolds number turbulence. A priori tests in isotropic turbulence and wall-bounded turbulence are performed. A posteriori tests of decaying turbulence and channel Poiseuille flow are made to testify the model performance, especially on the energy backscatter. The simple constant coefficient formulation has good performance, and avoids the ensemble average operation, which exists in other subgrid models. This constant improved VI model is particularly proposed in complicated large-eddy simulation projects. (C) 2009 American Institute of Physics. [DOI: 10.1063/1.3153911]
\end{abstract}

\section{INTRODUCTION}

In large-eddy simulation (LES), different subgrid stress models are already available. For instance, the well-known Smagorinsky model ${ }^{1}(\mathrm{SM})$ is the most popular "eddy viscosity" model. In the scale similarity model, ${ }^{2}$ another method based on the correlation between resolved and subgrid scales is utilized. Geurts ${ }^{3}$ employed an inverse model to improve the similarity models by mathematical method. Brun et l. $^{4}$ introduced a velocity increment model (VI model), which was inspired by structure function formulation in Refs. 5-7. In their work, the second-order velocity increment was compared to subgrid stress analytically and numerically. Their tensor properties were exactly the same. Besides, the anisotropy properties were analyzed in a priori tests of pipe flow, which was in good agreement with the exact direct numerical simulation (DNS) results. Compared to the classical eddy viscosity models, VI model could both simulate the forward and backward scatters. ${ }^{8-10}$ However, problems still remain in this model. For example, the model coefficient was not determined physically. Both Germano identity ${ }^{11}$ and another dynamic procedure based on Taylor expansion are introduced in this model, but they lead to quite different values in real calculation.

In addition to the mathematical methods, another way for subgrid modeling is to use the physical knowledge of the turbulence, i.e., the theory of multiscale energy transfer. In spectral space, energy transfer can be considered as a flux beyond the cutoff wave numbers in Kraichnan's theory. ${ }^{12}$ There are several subgrid-scale (SGS) models based on the transfer properties in spectral space, such as the effective viscosity model proposed by Chollet and Lesieur. ${ }^{13}$ In physical space, the model introduced by Cui et al. ${ }^{14}$ (CZZS model) is an eddy viscosity model based on the two-point energy transfer equation, namely, the Kolmogorov equation for filtered (KEF) velocity. Since VI model is a two-point model, we try to apply KEF to study the two-point energy transfer properties.

A filter size is defined as $\Delta=\pi / k_{c}$, where $k_{c}$ is the cutoff wave number in case of spectral cutoff filter. The superscripts $\bullet<$ and $\bullet>$ represent the grid-scale and subgrid-scale, parts respectively. The angle brackets $\langle\bullet\rangle$ represent an ensemble average. In numerical cases, this average operation is implemented by a spatial average in the homogeneous isotropic directions, instead. The subscript $l$ denotes a component in the direction of the two-point distance $\boldsymbol{\xi}$, and in isotropic turbulence we can also assume it to be in $x_{1}$ direction. The KEF in homogeneous isotropic turbulence with high Reynolds number could be described as ${ }^{15}$

$$
-\frac{4}{5} \epsilon_{f} \xi=D_{l l l}^{<}(\xi)-6 T_{l, l l}(\xi),
$$

where $\xi$ is independent with the filter size $\Delta$ of LES. Define the VI $\delta \mathbf{u}(\mathbf{x}, \boldsymbol{\xi})=\mathbf{u}(\mathbf{x}+\boldsymbol{\xi})-\mathbf{u}(\mathbf{x})$. On the right-hand side of Eq. (1), $D_{l l l}^{<}(\xi)=\left\langle\delta u_{1}^{<}(\xi) \delta u_{1}^{<}(\xi) \delta u_{1}^{<}(\xi)\right\rangle$ is the third-order longitudinal structure function, and $T_{l, l l}(\xi)=\left\langle u_{1}^{<}\left(x_{1}\right) \tau_{11}^{<}\left(x_{1}+\xi\right)\right\rangle$ is the subgrid energy transfer. The dissipation rate of subgridscale turbulence $\epsilon_{f}$ equals $-\left\langle\tau_{i j}^{<} S_{i j}^{<}\right\rangle$, where $\tau_{i j}^{<}=\left(u_{i} u_{j}\right)^{<}$ $-u_{i}^{<} u_{j}^{<}$and $S_{i j}^{<}=1 / 2\left(\partial u_{i}^{<} / \partial x_{j}+\partial u_{j}^{<} / \partial x_{i}\right)$. The dissipation term could be represented by using the 11 components in isotropic turbulence,

$$
-\frac{4}{5} \epsilon_{f}=6\left\langle\tau_{11}^{<} S_{11}^{<}\right\rangle .
$$

This equation is satisfied in isotropic turbulence. While in the applications of wall-bounded turbulence, the near-wall properties of subgrid model should be considered. It is well known that the classic SM causes a wrong near-wall behavior. Numerous studies are aiming at correcting the near-wall behavior of subgrid model. ${ }^{16,17}$ Lévêque et al. ${ }^{18}$ introduced an improved SM by considering the generalized Kolmogorov equation. ${ }^{19}$ This model represents better characters of scale separating for inhomogeneous shear flow and satisfies well the near-wall behavior. A more general way was employed to 
improve the CZZS model in homogeneous shear flow, with the consideration of mean velocity. ${ }^{20}$ The structure function is applied by using velocity fluctuation, accounting for the "local isotropic" $" 21,22$ as a slow part.

In this paper, we employ KEF to improve the VI model. In high Reynolds number turbulence, a constant model formulation of improved velocity increment model (IVI) is obtained. A priori and a posteriori tests are done in both homogeneous isotropic turbulence and channel Poiseuille flow. An equivalent eddy viscosity is utilized to check the nearwall behavior; the backscatter property is also checked. Results demonstrate that IVI model represents correct near-wall behavior and predicts well both forward and backward energy transfer. The improvements comparing with VI model are discussed particularly.

\section{IMPROVED VELOCITY INCREMENT MODEL}

Brun suggested that the subgrid stresses tensor could be modeled as

$$
\tau_{i j}^{<}(\mathbf{x}, \Delta)=C_{f}\left(\Delta^{\prime}\right) Q_{i j}\left(\mathbf{x}, \Delta^{\prime}\right),
$$

where $C_{f}$ is the dynamic model coefficient, depending on another distance $\Delta^{\prime}$. In this paper, the second-order VI tensor $Q_{i j}$ with a distance $\Delta^{\prime}$ is written as (without summation convention)

$$
\begin{aligned}
Q_{i j}\left(\mathbf{x}, \Delta^{\prime}\right)= & \frac{1}{2}\left[( u _ { i } ^ { < } ( \mathbf { x } + \Delta ^ { \prime } \mathbf { e } _ { i } ) - u _ { i } ^ { < } ( \mathbf { x } ) ) \left(u_{j}^{<}\left(\mathbf{x}+\Delta^{\prime} \mathbf{e}_{j}\right)\right.\right. \\
& \left.-u_{j}^{<}(\mathbf{x})\right)+\left(u_{i}^{<}(\mathbf{x})-u_{i}^{<}\left(\mathbf{x}-\Delta^{\prime} \mathbf{e}_{i}\right)\right)\left(u_{j}^{<}(\mathbf{x})\right. \\
& \left.\left.-u_{j}^{<}\left(\mathbf{x}-\Delta^{\prime} \mathbf{e}_{j}\right)\right)\right] .
\end{aligned}
$$

Although different from Brun's definition, it also satisfies the tensor symmetry

$$
Q_{i j}\left(\mathbf{x}, \Delta^{\prime}\right)=Q_{i j}\left(\mathbf{x},-\Delta^{\prime}\right), \quad Q_{i j}=Q_{j i} .
$$

The differences of the definitions are discussed in Sec. IV.

In order to be applied to KEF in homogeneous isotropic turbulence, the longitudinal component is particularly discussed,

$$
\begin{aligned}
Q_{11}\left(x_{1}, \Delta^{\prime}\right)= & \frac{1}{2}\left[\delta u_{1}^{<}\left(x_{1}-\Delta^{\prime}, \Delta^{\prime}\right) \delta u_{1}^{<}\left(x_{1}-\Delta^{\prime}, \Delta^{\prime}\right)\right. \\
& \left.+\delta u_{1}^{<}\left(x_{1}, \Delta^{\prime}\right) \delta u_{1}^{<}\left(x_{1}, \Delta^{\prime}\right)\right] .
\end{aligned}
$$

From Eqs. (2), (3), and (6), and isotropy assumption, the subgrid dissipation could be denoted as

$$
\begin{aligned}
-\frac{4}{5} \epsilon_{f} \xi & =6 C_{f}\left\langle Q_{11}\left(\Delta^{\prime}\right) S_{11}^{<}\right\rangle \xi \\
& =6 C_{f}\left\langle\delta u_{1}^{<}\left(\Delta^{\prime}\right) \delta u_{1}^{<}\left(\Delta^{\prime}\right) \frac{\partial u_{1}^{<}}{\partial x_{1}}\right\rangle \xi \\
& =6 C_{f}\left\langle\delta u_{1}^{<}\left(\Delta^{\prime}\right) \delta u_{1}^{<}\left(\Delta^{\prime}\right) \frac{\partial \delta u_{1}^{<}\left(\Delta^{\prime}\right)}{\partial \Delta^{\prime}}\right\rangle_{\xi} \\
& =\left.2 C_{f} \xi \frac{\partial D_{l l l}^{<}(r)}{\partial r}\right|_{r=\Delta^{\prime}},
\end{aligned}
$$

and the subgrid transfer term reads

$$
-6 T_{l, l l}(\xi)=-6 C_{f}\left\langle u_{1}^{<}\left(x_{1}\right) Q_{11}\left(x_{1}+\xi, \Delta^{\prime}\right)\right\rangle .
$$

Therefore, Eq. (1) could be written as

$$
\begin{aligned}
\left.2 C_{f} \xi \frac{\partial D_{l l l}^{<}(r)}{\partial r}\right|_{r=\Delta^{\prime}}= & D_{l l l}^{<}(\xi)-6 C_{f}\left\langle u_{1}^{<}\left(x_{1}\right)\right. \\
& \left.\times Q_{11}\left(x_{1}+\xi, \Delta^{\prime}\right)\right\rangle,
\end{aligned}
$$

and the model coefficient is

$$
C_{f}=\frac{D_{l l l}^{<}(\xi)}{\left.2 \xi \frac{\partial D_{l l l}^{<}(r)}{\partial r}\right|_{r=\Delta^{\prime}}+6\left\langle u_{1}^{<}\left(x_{1}\right) Q_{11}\left(x_{1}+\xi, \Delta^{\prime}\right)\right\rangle} .
$$

Three different scales implied in this result should be clarified: The physical quantities are filtered at a filter size $\Delta$, VI model assumes $C_{f}$ and $Q_{i j}$ at a distance $\Delta^{\prime}$, and the equation itself is based on a distance $\xi$. In the following parts, the three scales will be analyzed primarily in an ideal high Reynolds number turbulence, and then in a moderate Reynolds number flow.

\section{A. Model analysis in high Reynolds number turbulence}

In the inertial subrange of homogeneous isotropic turbulence, the Kolmogorov theory ${ }^{23}$ predicts that the scaling law of structure function is satisfied,

$$
F_{n}(r) \propto r^{n / 3}
$$

where $F_{n}(r)=\left\langle\left(u_{1}(r)-u_{1}(0)\right)^{n}\right\rangle$ is the $n$th order structure function of the nonfiltered velocity in longitude direction. However, in KEF we focus on the scaling law of structure functions of filtered velocity. From Appendix, we prove that when $\Delta \ll r$, the properties of second-order structure functions are approximately the same between filtered and nonfiltered velocities. Regarding third-order structure functions, the proving process is much more complex, but the same conclusion could be obtained by applying similar magnitude analysis. It means that the scaling law of filtered velocity will be satisfied when $\Delta \ll r$.

Therefore, by using scaling law, the first term of the denominator in Eq. (10) could be simplified when $\Delta \ll \Delta^{\prime}$ and $\Delta^{\prime}$ is in inertial subrange,

$$
\left.2 \xi \frac{\partial D_{l l l}^{<}(r)}{\partial r}\right|_{r=\Delta^{\prime}}=\frac{2 \xi}{\Delta^{\prime}} D_{l l l}^{<}\left(\Delta^{\prime}\right) .
$$

If $\xi$ is also in inertial subrange, we could obtain

$$
\left.2 \xi \frac{\partial D_{l l l}^{<}(r)}{\partial r}\right|_{r=\Delta^{\prime}}=2 D_{l l l}^{<}(\xi) .
$$

The second term of the denominator in Eq. (10) corresponds to the transfer term of KEF. According to Meneveau's analysis, ${ }^{15}$ it tends to zero when $\xi$ is large. In fact, it is a correlation function between velocity $u_{1}^{<}$and VI $Q_{11}$ at distance $\Delta^{\prime}$, thus it tends to zero when $\xi$ is large. From Eqs. (6) and (8), we could also easily evaluate the magnitude when $\Delta^{\prime} \ll \xi$, 


$$
\begin{aligned}
\left\langle u_{1}^{<}\left(x_{1}\right) Q_{11}\left(x_{1}+\xi, \Delta^{\prime}\right)\right\rangle & \sim\left\langle\delta u_{1}^{<}(\xi) \delta u_{1}^{<}\left(\Delta^{\prime}\right) \delta u_{1}^{<}\left(\Delta^{\prime}\right)\right\rangle \\
& \ll D_{l l l}^{<}(\xi) .
\end{aligned}
$$

Thus the transfer term in Eq. (10) could be neglected, and the coefficient is

$$
C_{f}=\frac{D_{l l l}^{<}(\xi)}{2 D_{l l l}^{<}(\xi)}=\frac{1}{2} .
$$

The value does not depend on $\xi$. It agrees with the model assumption (3), where $C_{f}$ is only a function of $\Delta$ and $\Delta^{\prime}$. Notice that this result is satisfied only when the inertial subrange is wide enough, and $\Delta^{\prime}$ and $\xi$ are both in inertial subrange. Besides, in order to neglect the molecular terms in original KEF (Ref. 14), the filter size of LES should be much larger than the dissipation scale $\eta$. Therefore, we could write the combined multiscale relation,

$$
\eta \ll \Delta \ll \Delta^{\prime} \ll \xi \ll L,
$$

where $L$ is the integrated scale.

\section{B. Model analysis in moderate Reynolds number turbulence}

In the numerical simulation of LES, the Reynolds number might not be high enough, and the inertial subrange is not wide enough. Equation (16) is difficult to be satisfied, thus we fix the scales as $\Delta=\Delta^{\prime}=\xi$, and simplify the form of VI model in another way. The subgrid energy transfer $T_{l, l l}$ could be represented by using VI. In homogeneous turbulence, it is only a function of $\xi$, and does not depend on the location $x_{1}$. It could be represented as

$$
\begin{aligned}
T_{l, l l}(\xi)= & \left\langle u_{1}^{<}\left(x_{1}\right) \tau_{11}^{<}\left(x_{1}+\xi\right)\right\rangle \\
= & C_{f}\left\langle u_{1}^{<}\left(x_{1}\right) Q_{11}\left(x_{1}, 2 \xi\right)\right\rangle \\
= & \frac{1}{2} C_{f}\left[\left\langle u_{1}^{<3}\right\rangle-2\left\langle u_{1}^{<}\left(x_{1}\right) u_{1}^{<}\left(x_{1}\right) u_{1}^{<}\left(x_{1}+\xi\right)\right\rangle\right. \\
& +2\left\langle u_{1}^{<}\left(x_{1}\right) u_{1}^{<}\left(x_{1}+\xi\right) u_{1}^{<}\left(x_{1}+\xi\right)\right\rangle \\
& +\left\langle u_{1}^{<}\left(x_{1}\right) u_{1}^{<}\left(x_{1}+2 \xi\right) u_{1}^{<}\left(x_{1}+2 \xi\right)\right\rangle \\
& \left.-2\left\langle u_{1}^{<}\left(x_{1}\right) u_{1}^{<}\left(x_{1}+\xi\right) u_{1}^{<}\left(x_{1}+2 \xi\right)\right\rangle\right] .
\end{aligned}
$$

The third-order moment of velocity $\left\langle u_{1}^{<3}\right\rangle=0$. The last term in the right-hand side of Eq. (17) could be proved to be zero in isotropic turbulence,

$$
\begin{aligned}
\left\langle u_{1}^{<}\right. & \left.\left(x_{1}\right) u_{1}^{<}\left(x_{1}+\xi\right) u_{1}^{<}\left(x_{1}+2 \xi\right)\right\rangle \\
& =\left\langle u_{1}^{<}\left(x_{1}-\xi\right) u_{1}^{<}\left(x_{1}\right) u_{1}^{<}\left(x_{1}+\xi\right)\right\rangle \\
& =-\left\langle u_{1}^{<}\left(x_{1}+\xi\right) u_{1}^{<}\left(x_{1}\right) u_{1}^{<}\left(x_{1}-\xi\right)\right\rangle \\
& =-\left\langle u_{1}^{<}\left(x_{1}\right) u_{1}^{<}\left(x_{1}+\xi\right) u_{1}^{<}\left(x_{1}+2 \xi\right)\right\rangle .
\end{aligned}
$$

Define the third-order correlations

$$
\begin{aligned}
& R_{l, l l}(2 \xi)=\left\langle u_{1}^{<}\left(x_{1}\right) u_{1}^{<}\left(x_{1}+2 \xi\right) u_{1}^{<}\left(x_{1}+2 \xi\right)\right\rangle, \\
& R_{l l, l}(2 \xi)=\left\langle u_{1}^{<}\left(x_{1}\right) u_{1}^{<}\left(x_{1}\right) u_{1}^{<}\left(x_{1}+2 \xi\right)\right\rangle .
\end{aligned}
$$

In isotropic turbulence $R_{l, l l}(2 \xi)=-R_{l l, l}(2 \xi)$. The subgrid energy transfer $T_{l, l l}$ reads

$$
T_{l, l l}(\xi)=-2 C_{f} R_{l l, l}(\xi)-\frac{1}{2} C_{f} R_{l l, l}(2 \xi) .
$$

Since in isotropic turbulence $D_{l l l}^{<}=6 R_{l l, l}$, the term of twopoint subgrid energy transfer

$$
T_{l, l l}(\xi)=-\frac{1}{3} C_{f} D_{l l l}^{<}(\xi)-\frac{1}{12} C_{f} D_{l l l}^{<}(2 \xi) .
$$

It is interesting to notice that the energy transfer has a linear relation with the third-order structure function. In Eqs. (7) and (21) there might be energy backscatter because of the third-order structure function $D_{l l l}^{<}$, which will be further analyzed in Sec. III.

However, notice that in CZZS model, the eddy viscosity subgrid dissipation is always positive. The subgrid energy transfer depends on the gradient of the second-order structure function, that is,

$$
T_{l, l l}(\xi)=\nu_{t} \frac{\partial D_{l l}^{<}(\xi)}{\partial \xi} .
$$

From the classical scaling law $\partial D_{l l}^{<} / \partial \xi>0$ and the eddy viscosity $\nu_{t}>0$, it implies $T_{l, l l}>0$ is purely dissipative.

From Eqs. (1), (7), and (21), the coefficient $C_{f}$ could be obtained,

$$
C_{f}=\frac{2 D_{l l l}^{<}(\xi)}{\left.4 \xi \frac{\partial D_{l l l}^{<}(r)}{\partial r}\right|_{r=\xi}-4 D_{l l l}^{<}(\xi)-D_{l l l}^{<}(2 \xi)} .
$$

In applications, if the scaling law $D_{l l l}^{<}(\xi) \propto \xi^{n}$ is satisfied, we could obtain

$$
\left.\frac{\partial D_{l l l}^{<}(r)}{\partial r}\right|_{r=\xi}=n \frac{D_{l l l}^{<}(\xi)}{\xi},
$$

finally the dynamic form of IVI model could be

$$
C_{f}=\frac{2 D_{l l l}^{<}(\xi)}{4(n-1) D_{l l l}^{<}(\xi)-D_{l l l}^{<}(2 \xi)} .
$$

When $\Delta, \Delta^{\prime}, \xi$ tend to zero, the scaling exponent $n \rightarrow 3$. Also we have $D_{l l l}^{<}(2 \xi)=8 D_{l l l}^{<}(\xi)$, thus the denominator tends to zero. It agrees with the conclusion of Meneveau ${ }^{15}$ that $-T_{l, l l}(\xi)=\left\langle\tau_{11}^{<} S_{11}^{<}\right\rangle \xi$ in small $\xi$. However, notice that $n$ depends on $\xi$, thus $n$ is not a constant, and we could not have the relation $D_{l l l}^{<}(2 \xi)=2^{n} D_{l l l}^{<}(\xi)$. In real practice, the scaling exponent $n$ could be calculated by any numerical difference methods. However, we suggest to fix $n=2.5$ in real practices since it is simple and numerical stable, and is verified in $a$ priori cases, which will be discussed in Sec. IV.

When $\Delta=\Delta^{\prime}=\xi$, the ideal inertial subrange condition (16) is not satisfied, thus we should use the dynamic model form (25) instead of the constant model form (15). However, the low-cost constant model form is more convenient in calculation. In order to check whether the constant model form could be applied approximately in this case, different model forms are then verified by a priori and a posteriori tests in Sec. III. 


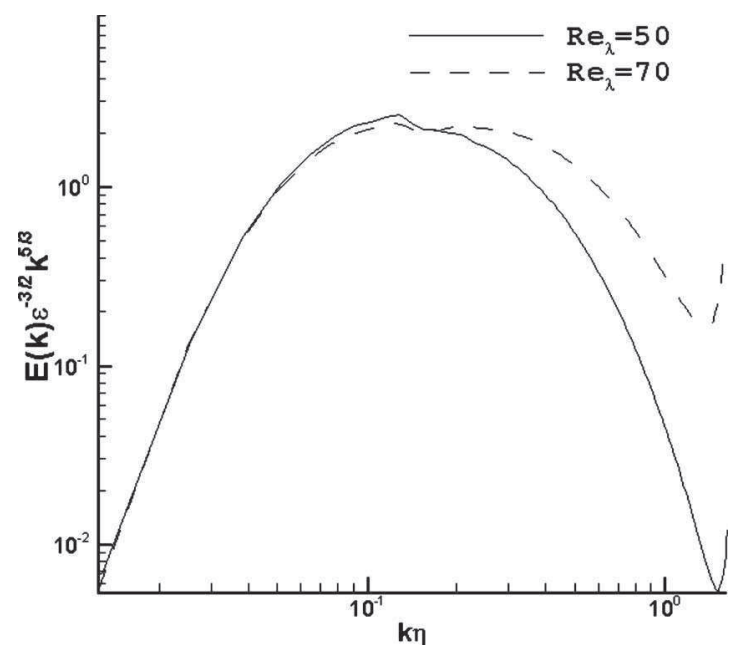

FIG. 1. The compensate energy spectrum in homogeneous isotropic turbulence. Solid line: $\operatorname{Re}_{\lambda}=50$; dashed line: $\operatorname{Re}_{\lambda}=70$.

\section{NUMERICAL VERIFICATIONS}

\section{A. A priori analysis}

Two DNS cases of homogeneous isotropic turbulence are used for a priori test. In these two cases, spectral method is applied, and a deterministic forcing method is employed to simulate a statistically stationary turbulence. The computation domain has $256^{3}$ grid. The grid size is denoted as $h$. Reynolds numbers $\operatorname{Re}_{\lambda}$ are 50 and 70, respectively. The compensate energy spectrums of DNS cases are shown in Fig. 1, where the plateaus represent the inertial subrange in spectral space. The corresponding wave number of the plateaus is about $0.1<k \eta<0.3$. The relevant filter size is $10<\Delta / \eta$ $<30$. In addition, the integrated scale $L \sim 70 \eta$ in the two cases, thus we could reasonably consider that $\eta \ll \Delta=\xi \ll L$. The plateau in the case $\operatorname{Re}_{\lambda}=70$ is wider than $\operatorname{Re}_{\lambda}=50$.

In homogeneous isotropic turbulence, the exact subgrid stress is calculated with different cutoff wave numbers. The coefficient of VI model is calculated as

$$
C_{f}=\frac{\left\langle\tau_{11}^{<} S_{11}^{<}\right\rangle}{\left\langle Q_{11} S_{11}^{<}\right\rangle} .
$$

The dynamic value of IVI model (25) is compared to the exact value (26) in Fig. 2, where the scaling exponent $n$ is calculated by using the first-order central difference method. It shows that the exact coefficient value increases with $\Delta$ and $\xi$, and it is about $1 / 2$ when $10<\xi / \eta \lesssim 18$, which is a small region of inertial subrange. Moreover, the dynamic model value is also in good agreement in this region. Therefore, in a practical LES case, if the filter size $\Delta$ and the two-point distance $\xi$ are fixed in this region, the constant form (15) of IVI model might be applied to obtain the low cost in calculation. When $\xi$ is small, the constant form is in disagreement. It stems from the effect of molecular viscosity, which is neglected in KEF. When $\xi$ is too large, the error is also obvious.

$\mathrm{KEF}$ is based on homogeneous isotropic turbulence, but in wall-bounded shear flow, the local isotropy could also be satisfied by considering the slow parts. ${ }^{14,22}$ In a local region,

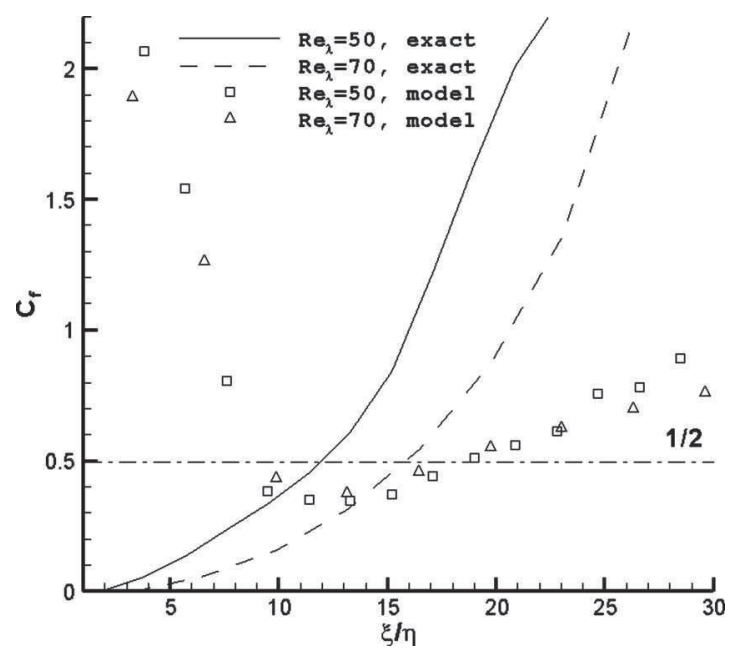

FIG. 2. Comparison between the exact value and dynamic model value of $C_{f}$, against different filter sizes and two-point distance $\Delta=\xi$, in homogeneous isotropic turbulence. Solid line with symbols: $\operatorname{Re}_{\lambda}=50$; dashed line with symbols: $\operatorname{Re}_{\lambda}=70$. The horizontal line is the theoretical value.

the two-point VI is considered as a slow part for subgrid modeling, which provides a good near-wall property. ${ }^{18} \mathrm{~A}$ DNS case of channel flow is used for a priori test. The pseudospectral method is applied. The Reynolds number $\operatorname{Re}_{H}=7000$, based on the bulk velocity $U_{m}$ and channel half width $H$. The Reynolds number based on friction velocity is approximated to 395 . The grid number is $128 \times 128 \times 64$. The computation domains are $4 \pi H, 2 H$, and $2 \pi H$ in streamwise, normal, and spanwise directions, respectively. The DNS grid size is denoted by $h$. The filter size of LES and the two-point distance in VI model are the same: $\Delta=\xi=2 h$. It is about 15 times of Kolmogorov scale, and can be treated as in inertial subrange.

In IVI model, the effective eddy viscosity $\nu_{t}$ could be defined as $\tau_{i j}^{<}=-2 \nu_{t} S_{i j}^{\prime<}$, in which the prime means the fluctuated part without mean flow. To compare with other models, it could be calculated as

$$
\nu_{t}=-C_{f} \frac{\left\langle Q_{i j}^{\prime} S_{i j}^{\prime<}\right\rangle}{2\left\langle S_{i j}^{\prime<} S_{i j}^{\prime<}\right\rangle},
$$

where $C_{f}$ is regarded as a constant $1 / 2$. The other SGS models used for comparison are the original SM with model constant $C_{s}=0.1$, the Germano dynamic Smagorinsky model (DSM), the SM with van Driest damping function in nearwall region (SM damping), and the CZZS model. A priori test of the magnitude of eddy viscosity is done, with the filter size $\Delta=2 h$. Results are shown in Fig. 3. The original SM causes a false viscosity at the wall, which does not tend to zero. An empirical method for solving this problem is to use van Driest damping function. An alternative mathematical way is the Germano dynamic model, which depends on the use of a test filter and a reference model. However, it does not fit well for the empirical location of peak $Y^{+} \simeq 25 .{ }^{18}$ In contrast, with KEF applied in SGS models, the near-wall property is naturally satisfied and the location of peak is well captured. 


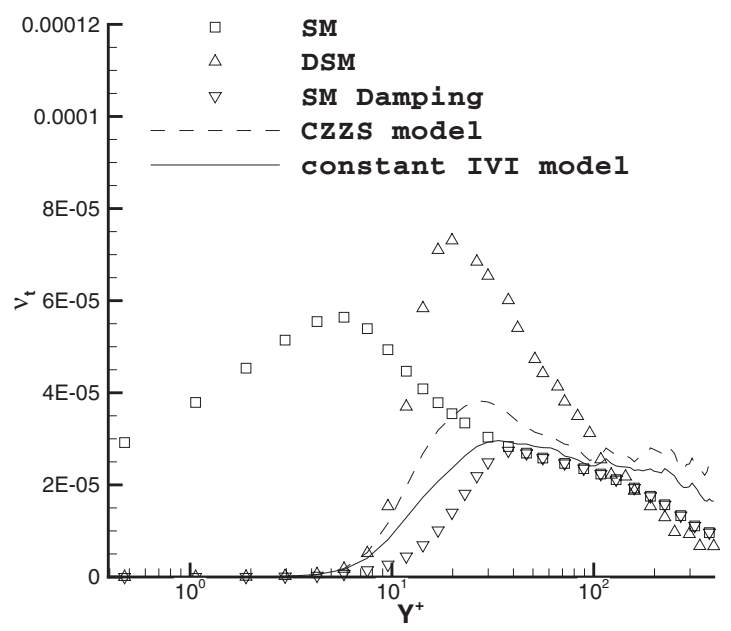

FIG. 3. A priori test of the eddy viscosity by using different SGS models in channel flow, with filter size $\Delta=2 h$. Symbols: SM with or without different corrections; lines: models based on KEF.

\section{B. Posteriori tests}

Two a posteriori tests are tried in this section. First, we employ IVI model in LES of homogeneous isotropic turbulence. Our LES cases correspond to the decay turbulence at low Reynolds number, similar to the results of Comte-Bellot experiment. ${ }^{24}$ These simulations are run on $48^{3}, 64^{3}$, and $96^{3}$ grids, respectively. Spectral method is applied in these cases. The initial fields are filtered from a full-developed forced turbulence. Figure 4 shows the spectrum in comparison with experiment data (the filled symbols) at $96 M$ and $172 M$. All cases are calculated with the constant coefficient, i.e., Eq. (15), and give satisfactory results.

Second, another attempt is done in channel flow. The parameters of Reynolds number, computation domain, and numerical method are the same as those in our DNS channel case. The grid numbers and subgrid models are shown in Table I. The DNS case is already introduced in the last section. The "no model case" applies the "DNS" method at a coarse grid $(32 \times 48 \times 32)$, which is compared to the LES

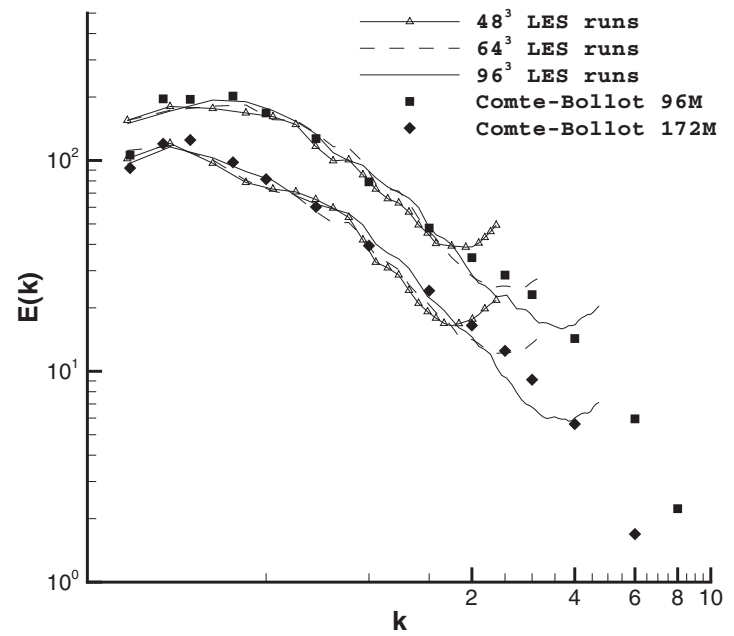

FIG. 4. Energy spectrum in decaying isotropic turbulence. Squares: ComteBellot 96M; diamonds: Comte-Bellot $172 M$; lines with triangles: $48^{3}$ LES runs; dashed lines: $64^{3}$ LES runs; solid lines: $96^{3}$ LES runs.
TABLE I. The parameters implemented in a posteriori tests.

\begin{tabular}{lcc}
\hline \hline Case & Grid & Model \\
\hline DNS case & $128 \times 128 \times 64$ & $\ldots$ \\
No model case & $32 \times 48 \times 32$ & $\ldots$ \\
Dynamic IVI model case & $32 \times 48 \times 32$ & Equation (25) \\
Constant IVI model case & $32 \times 48 \times 32$ & Equation (15) \\
\hline \hline
\end{tabular}

cases to show the model effect. Two LES cases are also executed at $32 \times 48 \times 32$ grid, with the dynamic IVI model formulation (25) and the constant IVI model formulation (15), respectively. The scaling exponent is given as $n=2.5$ in Eq. (25). A posteriori results are shown in Fig. 5.

In Fig. 5(a), the two SGS models both yield good agreement with DNS velocity profile, they are better than the "no model" case. In Figs. 5(b) and 5(c), the DNS results are filtered to the $32 \times 48 \times 32$ resolution, in order to be compared with others at the same grid scale. Both the two LES cases show quite better performances than the no model case. There are only slight differences between these two LES cases. The dynamic IVI model case is in slightly better agreement with the filtered DNS results in near-wall region. The peak locations of the kinetic energy and the Reynolds stress are a little better simulated in the dynamic IVI model case. However, the constant IVI model works quite well. Compared to other works, the results are as good as other dynamic subgrid models (the same channel flow were calculated using other models in Refs. 14 and 20), and all these models are much better than the original SM, especially in near-wall region. Therefore, the most valuable improvement in IVI model is not the accurate dynamic formulation, but is the constant coefficient introduced. It has as good performance as the other dynamic subgrid models, and the constant formulation is also greatly low cost. In addition, in the engineering projects of complicated geometries, it is difficult to calculate the structure functions using spatial average, but the constant coefficient value will be appropriate.

Further analysis is on the forward and backward flux in energy transfer. The SGS dissipation $\epsilon_{f}$ represents the energy transfer between resolved and subgrid scales. ${ }^{8}$ The contributions of the components of SGS dissipation were analyzed by Hartel et al., ${ }^{10}$ where the interactions between fluctuating stresses and fluctuating rates of strain are studied. From their analysis, the SGS energy is dissipated in streamwise and normal direction, and the backscatter of SGS energy is in spanwise direction. While $C_{f}$ is always positive, the nondimensionalized subgrid dissipation tensor components $\epsilon_{f i j}^{+}$ caused by fluctuations could be calculated as (without summation convention)

$$
\epsilon_{f i j}^{+}=\frac{-\left\langle\tau_{i j}^{\prime<} S_{i j}^{\prime<}\right\rangle}{\operatorname{rms}\left(\tau_{i j}^{\prime<}\right) \operatorname{rms}\left(S_{i j}^{\prime<}\right)}=\frac{-\left\langle Q_{i j}^{\prime} S_{i j}^{\prime<}\right\rangle}{\operatorname{rms}\left(Q_{i j}^{\prime}\right) \operatorname{rms}\left(S_{i j}^{\prime<}\right)} .
$$

The components of SGS dissipation are shown in Fig. 6. A priori results are from our DNS case of channel flow, and a posteriori results are from the constant IVI model case. In 


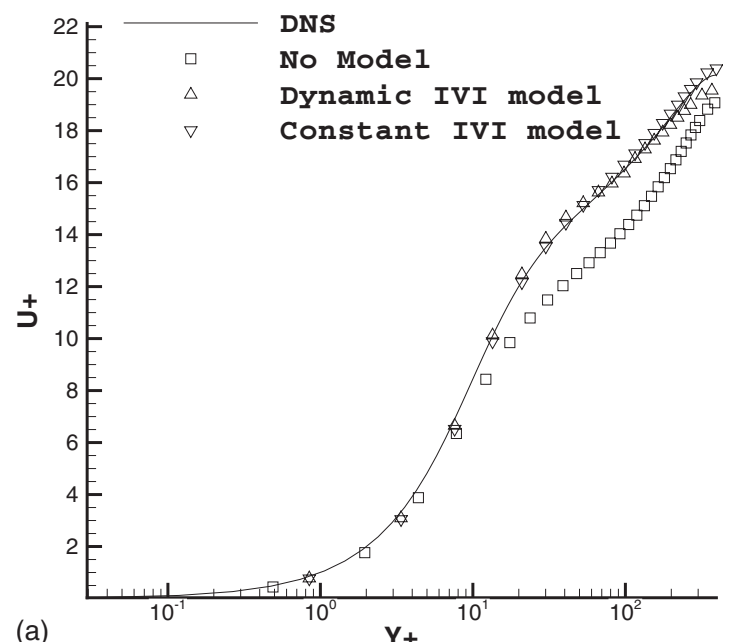

(a)

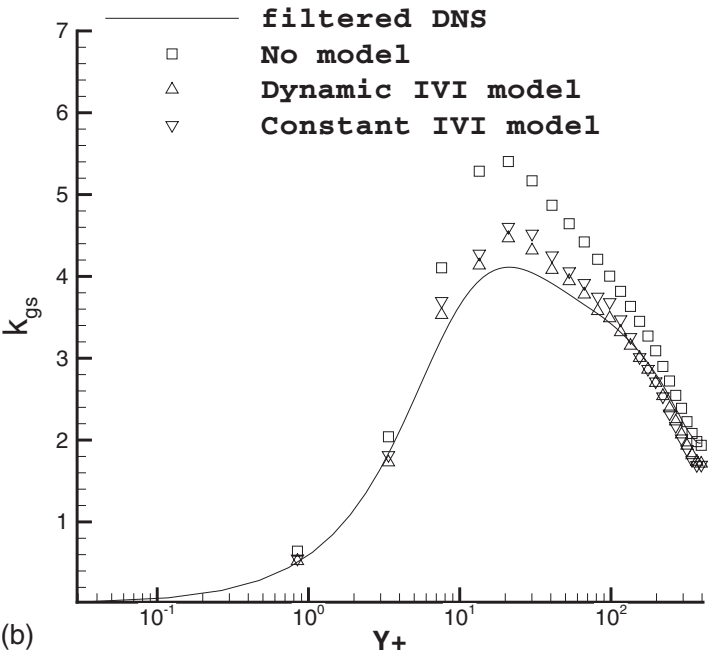

(b)

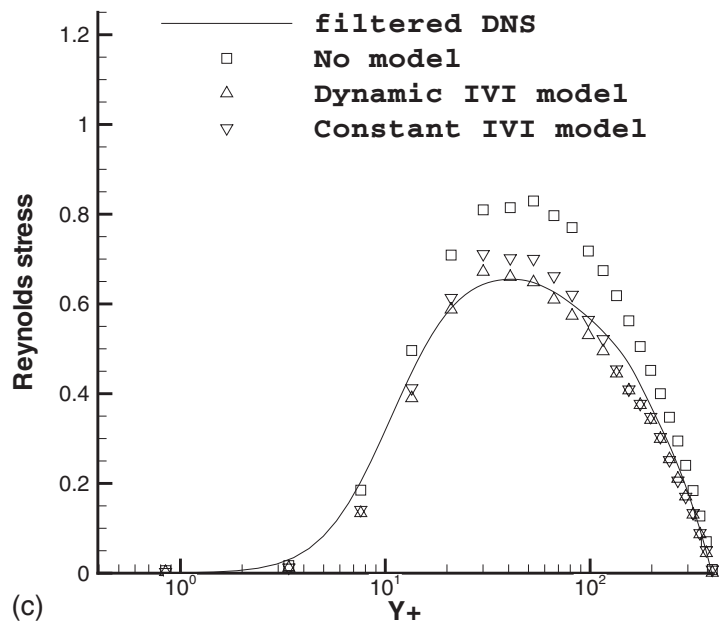

FIG. 5. A posteriori statistical results using different models (Table I) in channel flow. (a) Mean velocity. (b) Resolved-scale turbulence kinetic energy. (c) Resolved-scale Reynolds stress.

these figures, positive value means SGS energy dissipation, i.e., forward scatter; negative value represents the energy backscatter. Although the Reynolds numbers are different $\left(\operatorname{Re}_{\tau}=395\right.$ in our case and $\operatorname{Re}_{\tau}=180$ in the work of Hartel et al.), the results can be qualitatively compared. Among the normal components, $\boldsymbol{\epsilon}_{f 11}$ is the most important positive com-
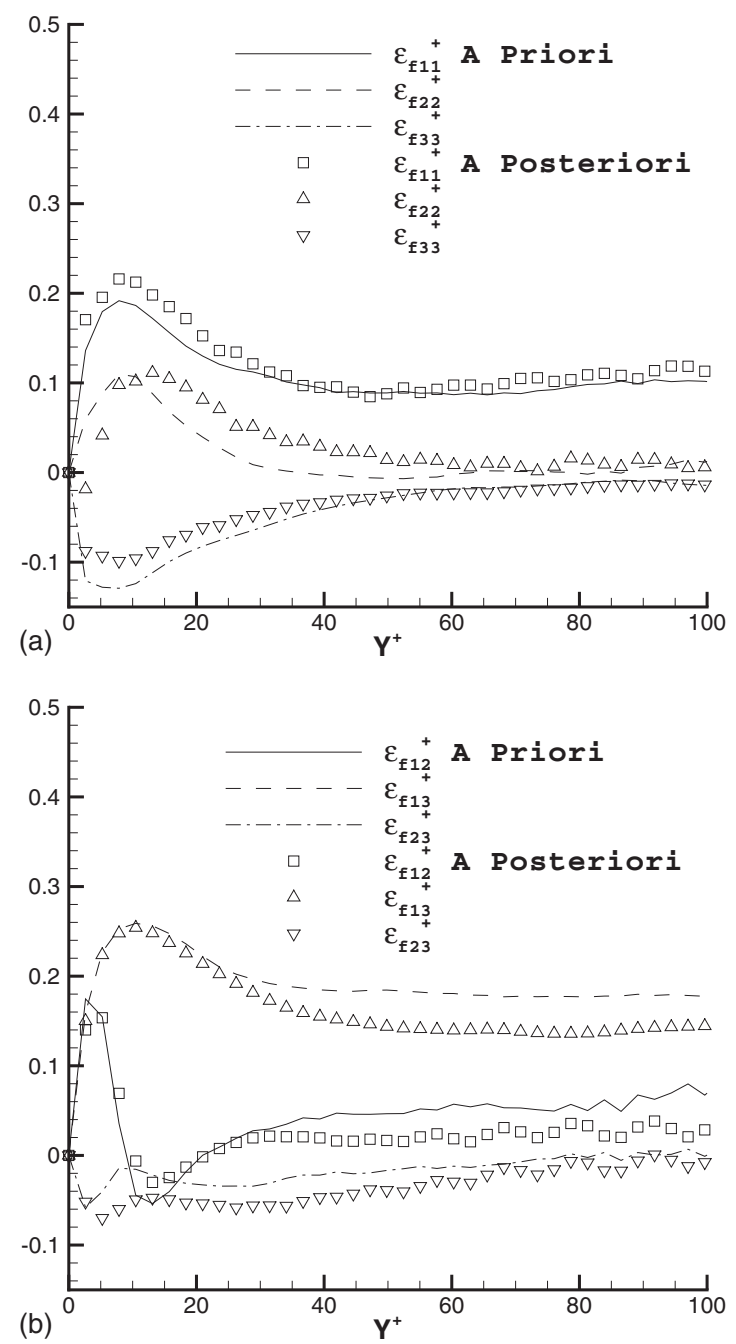

FIG. 6. The contributions of components for subgrid energy dissipation, normalized by using Eq. (28). Lines: a priori results; symbols: a posteriori results. (a) Normal components: $\epsilon_{f 11}^{+}, \epsilon_{f 22}^{+}$, and $\epsilon_{f 33}^{+}$. (b) Deviatonic components: $\epsilon_{f 12}^{+}, \epsilon_{f 13}^{+}$, and $\epsilon_{f 23}^{+}$.

ponent which leads to the dissipation, while the spanwise components $\epsilon_{f 33}$ causes a strong energy backscatter. Compared to Hartel's analysis (see Fig. 9 of Ref. 10), the disagreement only occurs in the $Y^{+}<8$ range, where the slight energy backscatter of $\epsilon_{f 11}$ is not shown by our model. It might be caused by the strong inhomogeneity in this region. Among the deviatonic components, the most special component is $\epsilon_{f 12}$, which has the backscatter in the region $10<Y^{+}<20$ and dissipates in the other ranges. This result is in good agreement with Hartel's analysis, including the peak locations. With each component, both a priori and a posteriori results assert the similar SGS dissipation. The backscatter properties of IVI model could stem from the third-order structure function. From Eqs. (7) and (21), the two-point energy dissipation and subgrid transfer are represented by the third-order structure function, which could have different values, either negative or positive, in different directions and in different regions. 


\section{DISCUSSION}

Before discussion, the different definitions of the VI $Q_{i j}$ should be clarified. Brun's original definition is based on the formulation of Taylor expansion, which contains both longitude and transverse components,

$$
\begin{aligned}
Q_{i j}\left(\mathbf{x}, \Delta^{\prime}\right)= & {\left[\sum_{k=1}^{3}\left[u_{i}^{<}\left(\mathbf{x}+\frac{1}{2} \Delta^{\prime} \mathbf{e}_{k}\right)-u_{i}^{<}\left(\mathbf{x}-\frac{1}{2} \Delta^{\prime} \mathbf{e}_{k}\right)\right]\right] } \\
& \times\left[\sum _ { k = 1 } ^ { 3 } \left[u_{j}^{<}\left(\mathbf{x}+\frac{1}{2} \Delta^{\prime} \mathbf{e}_{k}\right)\right.\right. \\
& \left.\left.-u_{j}^{<}\left(\mathbf{x}-\frac{1}{2} \Delta^{\prime} \mathbf{e}_{k}\right)\right]\right] .
\end{aligned}
$$

Comparing with it, the definition in IVI model, i.e., Eq. (4), is simplified without the transverse components. The main problem of this simplification is that the subgrid stress vanishes when the flow is locally characterized by a pure shear flow $u_{1}^{<}\left(x_{2}\right)$ somewhere. However, in this situation, the effect of dissipation are the same between definitions (4) and (29), both of them yield the zero subgrid dissipation $-\tau_{i j}^{<} S_{i j}^{<}=0$. This behavior can also be explicitly achieved in the Clark model. ${ }^{25}$

In the KEF formulation (1), all terms can be represented by the longitude components. Thus it could be appropriate to characterize the energy transfer using the longitude components, in homogeneous isotropic turbulence. Therefore, our simplified definition (4) can be employed. It is easy to implement Eq. (4) in IVI model with the constant coefficient.

It should be mentioned that the finite difference form of Eq. (29) is quite similar as the Clark model. However, Brun's work verified that the VI formulation can dissipate enough, ${ }^{4}$ which is much better than the poor dissipation effect of Clark model. The improvement may stem from the fact that more information is contained in the VI than in the velocity gradient, i.e., the high-order velocity derivatives.

In addition to the definition of VI $Q_{i j}$, the most important improvement in IVI model is to determine the model coefficient $C_{f}$ by employing the two-point energy transfer equation, i.e., KEF. The dynamic procedure proposed by Brun is based on Taylor expansion, it can be written as

$$
C_{f}=\frac{1}{\alpha^{2}} \frac{L_{k k}(\alpha)}{Q_{i i}},
$$

in which $L_{i j}(\alpha)=\widehat{u_{i}^{<} u_{j}^{<}}-\widehat{u_{i}^{<}} \widehat{u_{j}^{<}}$is the Leonard stress and $\hat{\bullet}$ is the test filter at size $\alpha \Delta$.

Another method introduced by Brun is to employ the Germano identity $L_{i j}(\alpha)=C_{f}\left(Q_{i j}(\Delta, \alpha \Delta)-Q_{i j}(\Delta, \Delta)\right)$, by considering $C_{f}$ as a constant. The coefficient can be then represented as

$$
C_{f}=\frac{L_{i i}(\alpha)}{Q_{k k}(\Delta, \alpha \Delta)-Q_{k k}(\Delta, \Delta)} .
$$

Although these methods are introduced with definition (29), they can also be applied with our definition (4). A priori tests using different methods are shown in Fig. 7. The filter size is selected as $\Delta=2 h$, i.e., the LES mesh has $64 \times 64$
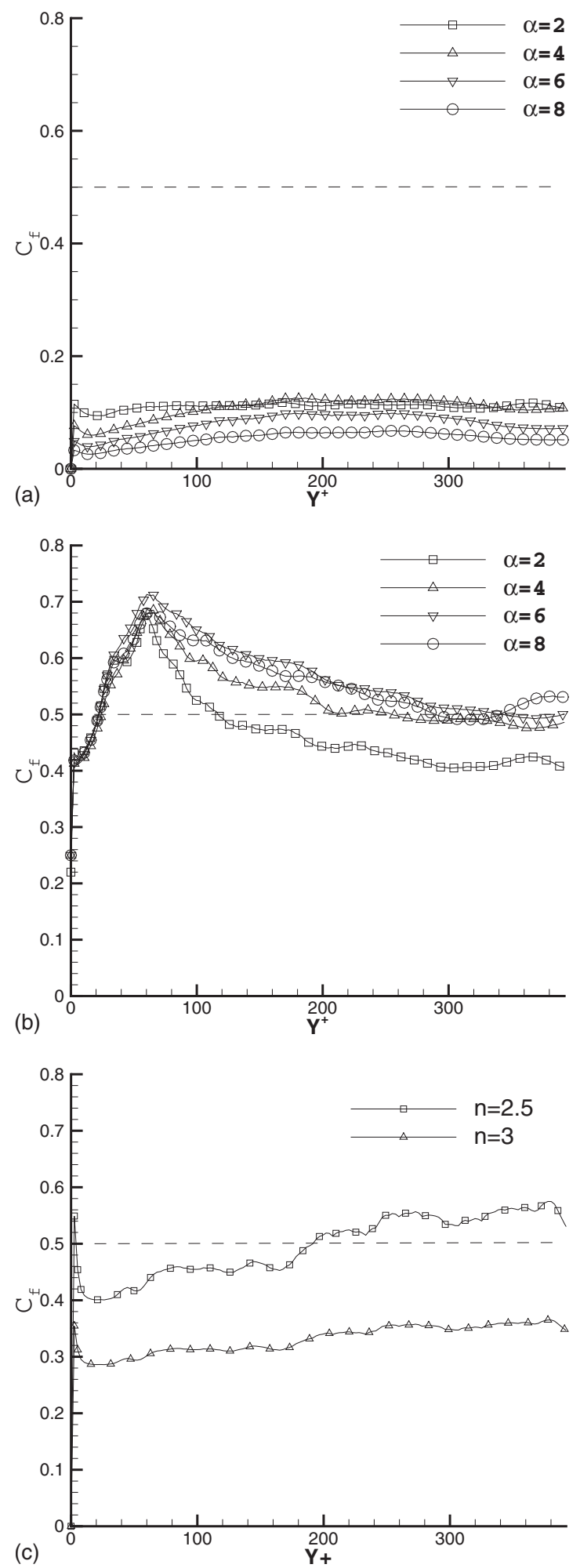

FIG. 7. A priori coefficient values using different dynamic methods, in channel flow. Filter size $\Delta=2 h$. (a) Based on Taylor expansion (30), with different test filters. (b) Based on Germano identity (31), with different test filters. (c) Dynamic formulation of IVI model (25), giving the scaling exponent $n=2.5$ and 3 . The dashed line is the constant value in IVI model.

$\times 32$ grids. The values using Taylor expansion are much smaller than other methods since Taylor expansion cannot be employed in large two-point distance. Germano identity yields $C_{f} \approx 0.5$ in the middle of the channel, which is in 
agreement with the dynamic IVI model when $n=2.5$. In the near-wall region, differences exist since the anisotropic effect is strong. However, the constant value 0.5 is also acceptably satisfied. In addition, $n=3$ yields much smaller value than 0.5 , and $n=2.5$ is in better agreement. That is the reason we propose $n=2.5$ in numerical calculations when utilizing the dynamic formulation (25).

A posteriori results in channel flow are shown in Fig. 8, in which the model coefficients are calculated by employing different methods. The parameters in this case are the same as described in the last section. From the figures, both Germano identity and the constant IVI model are better than the method based on Taylor expansion. Both of them are in good agreement with the DNS results, the constant coefficient is as good as the dynamic coefficient using Germano identity. However, the constant value is much less lost and easy to be implemented.

\section{CONCLUSION}

In conclusion, the VI model is improved by introducing $\mathrm{KEF}$ in this paper. The IVI model coefficient, solved dynamically, is proved to be a constant in high Reynolds number turbulence. This constant value satisfies the classical scaling law and demonstrates the two-point energy transfer properties. Compared to CZZS model, the difference relies on the subgrid energy transfer term $T_{l, l l}$. It is described by the second-order structure function in CZZS model, but connected with the third-order structure function in IVI model. Thus, the energy backscatter property could be represented in IVI model.

A priori verification in homogeneous isotropic turbulence shows that the constant form of IVI model could be approximately satisfied when both $\Delta$ and $\xi$ are in inertial subrange. The later applications in wall-bounded flow indicate the properties in near-wall region, respectably agreeing with the results derived from the empirical and Germano dynamic method for wall correction. The near-wall behaviors of IVI model stem from the properties of KEF, which only contain the homogeneous, local isotropic information in modeling. It was proved quite useful in LES of shear flow by Lévêque et al. ${ }^{18}$ Besides, a priori and a posteriori tests show the energy backscatter of different components of SGS dissipation, in good agreement with the results of Hartel et al. Compared to DNS results, a posteriori performances in both homogeneous isotropic flow and channel flow are also satisfactory. The constant formulation is as good as the dynamic formulation.

Comparing with the original VI model introduced by Brun et al., two differences are discussed. First, the formulation of $Q_{i j}$ is simplified. It retains only the longitude components, but does not change the dissipation properties. Second, which is the most important, the model coefficient can be determined as a simple constant. It can provide as good results as Germano identity. It is greatly low cost, and it avoids the ensemble average operator, which is difficult to be implemented in complicated flow. Therefore, we propose this constant IVI model in particular. It satisfies the two-point
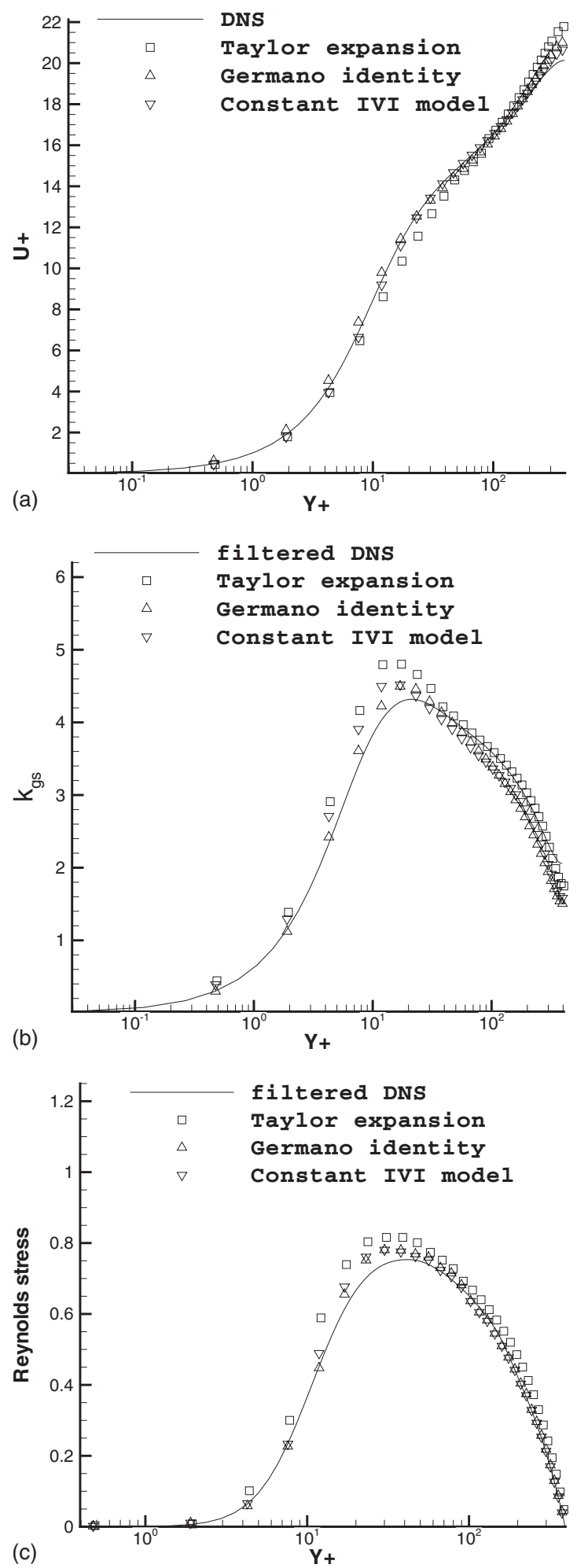

FIG. 8. A posteriori statistical results of channel flow, the model coefficient is determined by different methods. Filter size $\Delta=2 h$. (a) Mean velocity. (b) Resolved-scale turbulence kinetic energy. (c) Resolved-scale Reynolds stress.

energy equation (KEF), and implies the scaling law in inertial subrange. It is low cost in calculation, and easy to be implemented. It could be promising when applied in engineering projects. 


\section{ACKNOWLEDGMENTS}

L. Shao wishes to express the sincere gratitude to the support from the NSFC (Grant Nos. 10828204 and A020401) and BUAA SJP 111 program. L. Fang was supported by the Chinese Scholarship Council and LIAMA97-03.

\section{APPENDIX: RELATION BETWEEN SECOND-ORDER STRUCTURE FUNCTIONS OF FILTERED AND NONFILTERED VELOCITIES}

A filter in physical space is represented by introducing the kernel

$$
\int G\left(\mathbf{x}-\mathbf{x}^{\prime}\right) d \mathbf{x}^{\prime}=1,
$$

and the VI $\delta u_{i}^{<}(\mathbf{x})=u_{i}^{<}(\mathbf{x}+\boldsymbol{\xi})-u_{1}^{<}(\mathbf{x})$ could be written as

$$
\delta u_{i}^{<}(\mathbf{x})=\int G\left(\mathbf{x}-\mathbf{x}^{\prime}\right) \delta u_{i}\left(\mathbf{x}^{\prime}\right) d \mathbf{x}^{\prime} .
$$

Following Germano, ${ }^{26}$ it is easy to verify that

$$
\begin{aligned}
\delta u_{i}^{<} \delta u_{j}^{<}= & \left(\delta u_{i} \delta u_{j}\right)^{<} \\
& -\frac{1}{2} \iint G\left(\mathbf{x}-\mathbf{x}^{\prime}\right) G\left(\mathbf{x}-\mathbf{x}^{\prime \prime}\right)\left(u_{i}\left(\mathbf{x}^{\prime}\right)-u_{i}\left(\mathbf{x}^{\prime \prime}\right)\right) \\
& \times\left(u_{j}\left(\mathbf{x}^{\prime}\right)-u_{j}\left(\mathbf{x}^{\prime \prime}\right)\right) d \mathbf{x}^{\prime} d \mathbf{x}^{\prime \prime} .
\end{aligned}
$$

Using the same coordinate transformation as Germano, and assuming that the filter is Gaussian with filter size $\Delta$, finally we could obtain

$$
\begin{aligned}
\left\langle\delta u_{i}^{<} \delta u_{j}^{<}\right\rangle= & \left\langle\left(\delta u_{i} \delta u_{j}\right)^{<}\right\rangle-\frac{1}{2} \iint G(\mathbf{s} ; \sqrt{2} \Delta)\left\langle\left(\delta u_{i}(\mathbf{s})\right.\right. \\
& \left.\left.-\delta u_{i}(\mathbf{0})\right)\left(\delta u_{j}(\mathbf{s})-\delta u_{j}(\mathbf{0})\right)\right\rangle d \mathbf{s} .
\end{aligned}
$$

Because of the effect of filter kernel $G(\mathbf{s} ; \sqrt{2} \Delta)$, the last term of Eq. (A4) is mostly effective when $s \lesssim \Delta$. When $\Delta \ll r$, the production of the VIs in the last term could be evaluated that

$$
\begin{aligned}
& \left\langle\left(\delta u_{i}(\mathbf{s})-\delta u_{i}(\mathbf{0})\right)\left(\delta u_{j}(\mathbf{s})-\delta u_{j}(\mathbf{0})\right)\right\rangle \\
& \quad \ll\left\langle\left(\delta u_{i}(\mathbf{r})-\delta u_{i}(\mathbf{0})\right)\left(\delta u_{j}(\mathbf{r})-\delta u_{j}(\mathbf{0})\right)\right\rangle .
\end{aligned}
$$

The right-hand side is at most the same order of magnitude as $\left\langle\left(u_{i}(\mathbf{r})-u_{i}(\mathbf{0})\right)\left(u_{j}(\mathbf{r})-u_{j}(\mathbf{0})\right)\right\rangle$, i.e., $\left\langle\delta u_{i} \delta u_{j}\right\rangle$, it could be then estimated approximately,

$$
\left\langle\left(\delta u_{i}(\mathbf{s})-\delta u_{i}(\mathbf{0})\right)\left(\delta u_{j}(\mathbf{s})-\delta u_{j}(\mathbf{0})\right)\right\rangle \ll\left\langle\delta u_{i} \delta u_{j}\right\rangle .
$$

It can be neglected comparing with the first term in the righthand side of Eq. (A4).

In homogeneous turbulence, the first term in the righthand side of Eq. (A4) is

$$
\left\langle\left(\delta u_{i} \delta u_{j}\right)^{<}\right\rangle=\left\langle\delta u_{i} \delta u_{j}\right\rangle^{<}=\left\langle\delta u_{i} \delta u_{j}\right\rangle .
$$

Therefore when $\Delta \ll r$, there is the approximate relation that

$$
\left\langle\delta u_{i}^{<} \delta u_{j}^{<}\right\rangle \approx\left\langle\delta u_{i} \delta u_{j}\right\rangle .
$$

${ }^{1}$ J. Smagorinsky, "General circulation experiments with primitive equation," Mon. Weather Rev. 91, 99 (1963).

${ }^{2}$ J. Bardina, J. Ferziger, and W. C. Reynolds, "Improved subgrid-scale models for large-eddy simulation," AIAA Paper 80-1357, 1980.

${ }^{3}$ B. J. Geurts, "Inverse modeling for large-eddy simulation," Phys. Fluids 9, 3585 (1997)

${ }^{4}$ C. Brun, R. Friedrich, and C. B. da Silva, "A non-linear SGS model based on the spatial velocity increment," Theor. Comput. Fluid Dyn. 20, 1 (2006).

${ }^{5}$ O. Métais and M. Lesieur, "Spectral large-eddy simulation of isotropic and stably stratified turbulence," J. Fluid Mech. 239, 157 (1992).

${ }^{6} \mathrm{~J}$. O'Neil and C. Meneveau, "Subgrid-scale stresses and their modelling in a turbulent plane wake," J. Fluid Mech. 349, 253 (1997).

${ }^{7}$ R. J. Hill, “Applicability of Kolmogorov's and Monin's equations of turbulence," J. Fluid Mech. 353, 67 (1997).

${ }^{8}$ U. Piomelli, W. H. Cabot, P. Moin, and S. Lee, "Subgrid backscatter in turbulent and transitional flows," Phys. Fluids A 3, 1766 (1991).

${ }^{9}$ J. A. Domaradzki and E. M. Saiki, "A subgrid-scale model based on the estimation of unresolved scales of turbulence," Phys. Fluids 9, 2148 (1997).

${ }^{10}$ C. Hartel, L. Kleiser, F. Unger, and R. Friedrich, "Subgrid-scale energy transfer in the near-wall region of turbulent flows," Phys. Fluids 6, 3130 (1994).

${ }^{11}$ M. Germano, U. Piomelli, P. Moin, and W. H. Cabot, “A dynamic subgridscale eddy viscosity model," Phys. Fluids A 3, 1760 (1991).

${ }^{12}$ R. H. Kraichnan, "Inertial ranges in two-dimensional turbulence," Phys. Fluids 10, 1417 (1967).

${ }^{13}$ J. P. Chollet and M. Lesieur, "Parametrization of small scales of threedimensional isotropic turbulence utilizing spectral closures," J. Atmos. Sci. 38, 2747 (1981).

${ }^{14}$ G. X. Cui, H. B. Zhou, Z. S. Zhang, and L. Shao, "A new dynamic subgrid eddy viscosity model with application to turbulent channel flow," Phys. Fluids 16, 2835 (2004).

${ }^{15}$ C. Meneveau, "Statistics of turbulence subgrid-scale stresses: Necessary conditions and experimental tests," Phys. Fluids 6, 815 (1994).

${ }^{16}$ F. Nicoud and F. Ducros, "Subgrid-scale stress modelling based on the square of the velocity gradient tensor," Flow, Turbul. Combus. 62, 183 (1999).1386-6184

${ }^{17}$ E. R. van Driest, "On turbulent flow near a wall," AIAA J. 41, 259 (2003).

${ }^{18}$ E. Lévêque, F. Toschi, L. Shao, and J. P. Bertoglio, "Shear-improved Smagorinsky model for large-eddy simulation of wall-bounded turbulent flows," J. Fluid Mech. 570, 491 (2007).

${ }^{19}$ R. Benzi, G. Amati, C. Casciola, F. Toschi, and R. Piva, "Intermittency and scaling laws for wall bounded turbulence," Phys. Fluids 11, 1284 (1999).

${ }^{20}$ G. X. Cui, C. X. Xu, L. Fang, L. Shao, and Z. S. Zhang, "A new subgrid eddy-viscosity model for large-eddy simulation of anisotropic turbulence," J. Fluid Mech. 582, 377 (2007).

${ }^{21}$ U. Schumann, "Subgrid scales model for finite difference simulation of turbulent flows in plane channels and annuli," J. Comput. Phys. 18, 367 (1975).

${ }^{22}$ L. Shao, S. Sarkar, and C. Pantano, "On the relationship between the mean flow and subgrid stresses in large eddy simulation of turbulent shear flows," Phys. Fluids 11, 1229 (1999).

${ }^{23}$ A. N. Kolmogorov, "The local structure of turbulence in incompressible viscous fluid for very large Reynolds number," Proc. R. Soc. Edinburgh, Sect. A: Math. Phys. Sci. 30, 301 (1941).

${ }^{24}$ G. Comte-Bellot and S. Corrsin, "The use of contraction to improve the isotropy of grid generated turbulence," J. Fluid Mech. 25, 657 (1966).

${ }^{25}$ B. Vreman, B. Geurts, and H. Kuerten, "Large-eddy simulation of the temporal mixing layer using the Clark model," Theor. Comput. Fluid Dyn. 8, 309 (1996).

${ }^{26} \mathrm{M}$. Germano, "A direct relation between the filtered subgrid stress and the second order structure function," Phys. Fluids 19, 038102 (2007). 